\title{
APPLICATION OF THERMOELECTRIC MODULES AS RENEWABLE ENERGY SOURCES
}

\author{
Ivan ŠUMIGA, Dunja SRPAK, Živko KONDIĆ
}

\begin{abstract}
Thermoelectric modules are used for heating or cooling if an electric current flows through them. If one side of such a module is heated and the other side is cooled, the outputs show a potential difference that creates the current flow through an enclosed circuit. The paper describes briefly the physical basis of the operation of semiconductor thermoelectric modules and their application for generating electricity as thermoelectric generators. The experimental measurements were made with different available modules of thermoelectric generators. The analysis of obtained results has provided the conclusions about the possibility of their application as sources of electricity. Although the efficiency of thermoelectric modules is still quite low, the latest research reveals the possibility of the use of materials that make modules more efficient. Considering the fact that they do not contain moving parts, they are characterized by reliable, long-lasting work and simple operation and maintenance.
\end{abstract}

Keywords: electricity generation; materials; Peltier's cell; reliability; renewable energy sources; thermoelectric module

\section{INTRODUCTION}

Thermoelectric modules are often used for heating or cooling, but they can be used as generators as well, if one side of this module is heated and the other one is cooled. The basic concept of the thermoelectric theory is described in [1], as well as the experimental techniques helpful in the research of new thermoelectric materials.

The status of material development and thermo-electric applications is reviewed in [2]. The potential of achieving the environmental and economic benefits with thermoelectric applications in different areas are presented there, concluding the usability for micro/small applications, with the current conversion efficiency. However, using thermoelectrics in medium/large scale applications would be possible with better thermo-electric materials.

Different mechanical and electrical structures were explored in [3] to find the optimal solution to apply thermoelectric generators in low temperature resources, such as on thermal and geothermal sites or facilities where thermal energy is a by-product. Testing with different materials for thermoelectric generators in geothermal resources is also discussed in [4]. The evaluation of the manufacturability of thermoelectric devices combined with the total cost of system components is described in [5] in various applications for the use of the waste heat. Different vehicular applications are analyzed in [6] in order to reduce energy losses. Finding the optimal solution for the use of thermoelectric generators in automobiles was studied in [7-9] in order to recover the waste heat from the exhaust. As a heat source for thermoelectric generators, heat pipes were tested in [10] and the solar radiation in [11].

Theoretical analyses and experimental research as presented in [12-15] seek for better materials and structures that can improve the efficiency of thermoelectric modules.

This research examines the usability of market available thermoelectric modules with different prices but with similar technical characteristics. A comparison of the achieved electrical values will demonstrate the profitability of applying the tested modules in the use of waste heat from industrial machines.

The paper is organized as follows. First, the physical basis of the operation of semiconductor thermoelectric modules is described. Then, the possibility of their application for generating electricity is analyzed. The results of experimental measurements on several available thermogenerator modules are presented afterwards. The analysis of the obtained results, as well as the conclusions, give an overview of the possibilities of applying the Peltier modules as thermoelectric generators.

\section{DESCRIPTION OF THERMOELECTRIC MODULES}

If the electric conductive material is heated at one end, the electrons on that warmer part have higher kinetic energy than those at the colder part. Therefore, it comes to the electron diffusion from the warmer end to the colder end, i.e. the electrons are going to the state of lower temperature. Due to the uneven distribution of electrons, an electric field is formed, with a positive pole on the warmer part and negative pole on the colder part of the wire. The difference in potentials is created at the ends of the wire, which represent the source of the electromotive force.

If the connection point of two different metals that form an electrical circuit is heated, (point B in Fig. 1), the $U_{o}$ voltage will appear between the $\mathrm{T} 1$ and $\mathrm{T} 2$ points. That voltage is known as Seebeck's electromotive force. If resistance is connected between $\mathrm{T} 1$ and $\mathrm{T} 2$, the electrical current will flow through it. The compound acts as a thermoelectric voltage generator.

On the other hand, if the $U_{\text {in }}$ voltage is connected between $\mathrm{T} 1$ and $\mathrm{T} 2$, current will flow in that closed loop. At the junction point $\mathrm{A}$ of two metals, the cooling effect occurs because of the current flow; while at the junction point $\mathrm{B}$, the heating effect appears (Fig. 2). By changing the polarity of the voltage, the current flow is also changed. That results with the cooling in point B and heating in point A. Herein, the amount of heat absorbed at the cold end is equal to the heat released at the warm end. 


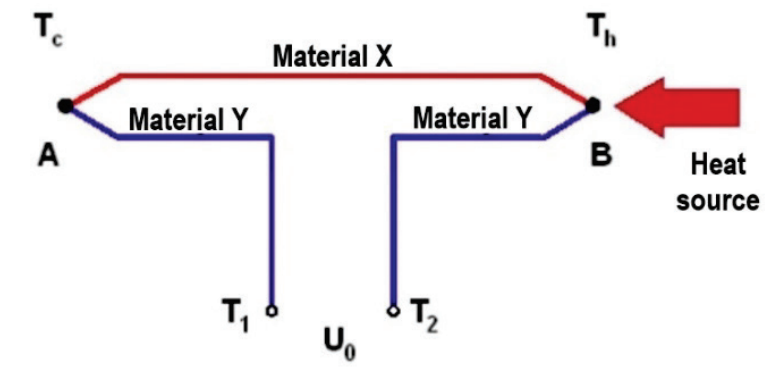

Figure 1 Seebeck effect [16]

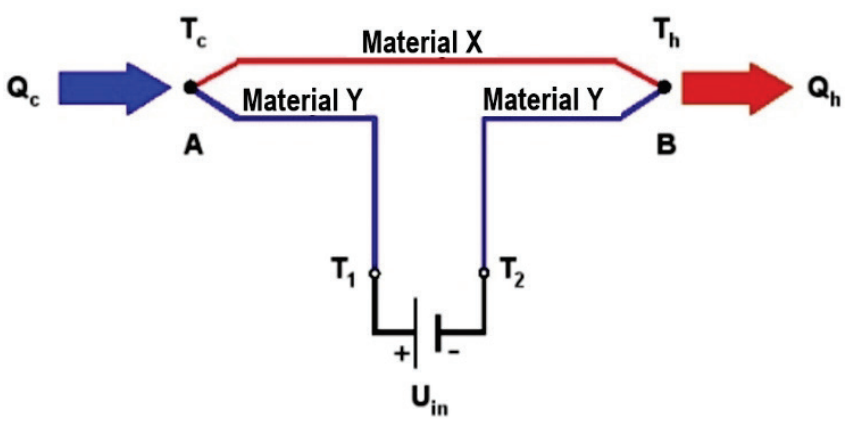

Figure 2 Peltier effect [16]

This phenomenon was first described by the French physicist Jean Peltier; therefore, it is called the Peltier effect. By applying the described phenomenon, a thermoelectric power generator or a heating or cooling device can be realized. The use of semiconductors instead of metals brings significant progress in the realization of thermoelectric modules. The realization of the thermoelectric module with semiconductors is shown in Fig. 3.

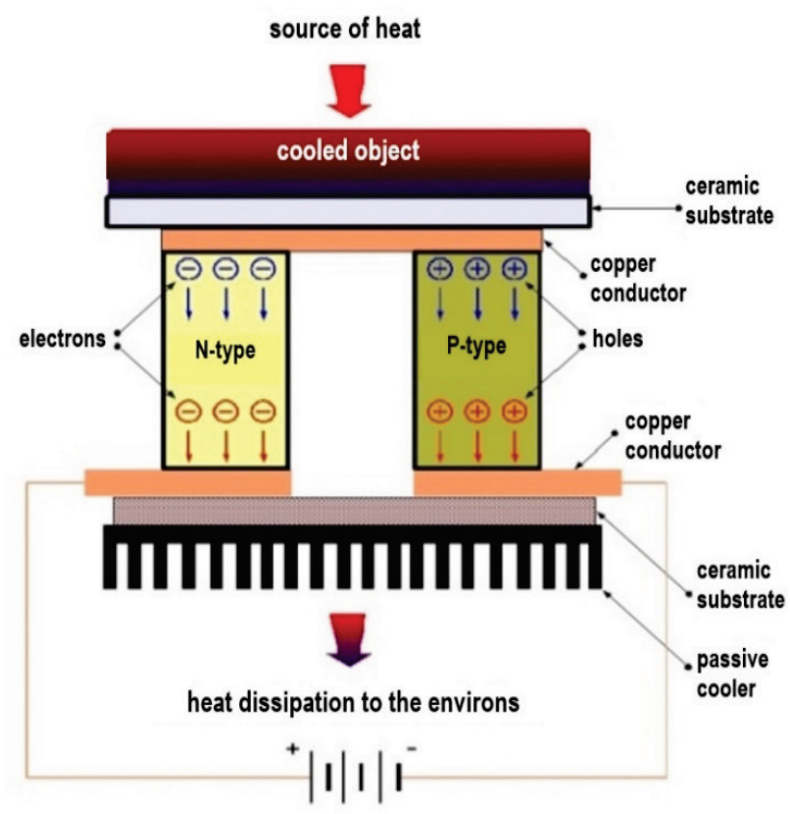

Figure 3 Thermoelectric modules for heating / cooling [16]

The free electrons in the copper conductor are at a higher energy level than the holes in the p-type semiconductor. Therefore, electrons release a part of the heat energy when passing from the copper conductor to the semiconductor. In the p-type semiconductor, electrons are recombined with the holes that move in the valence energy field of the semiconductor in the direction opposite of the direction of electron shifting. Since the p-type semiconductor is at a lower energy level than the conductor, at the next transition point, from the p-type semiconductor to the copper conductor, the electrons have to absorb the needed energy, due to which this joint is colder.

The next join is the connection of the cold conductor, the n-type semiconductor having a bottom of the conductive band at the higher energy level than Fermi level of the conductor. The electrons need to re-absorb enough heat energy to pass into the conductive band of the n-type semiconductor, which is why the join is colder. Through the n-type semiconductor, electrons move in a conductive energy band to the next connection of metals and semiconductors. Since free electrons in the conductive energy band of semiconductors are at a higher energy level than the Fermi level of the metal, at the transition to metal, they lose energy. Therefore, this joint is warmer than the environment.

Since no juncture of metal and semiconductor is a rectifier, by alteration of the polarity of external voltage, the side of the module used for cooling can also perform heating.

In order to obtain the higher heating or cooling power, more of the basic modules can be connected in series. Thus, the Peltier's heat pump is made, and is assembled of a large number of alternately arranged basic elements between two ceramic layers (Fig. 4). The heating or cooling capacity of such a heat pump depends on geometric dimensions, the number of the p-type and n-type semiconductor pairs, as well as on the properties of used materials. Unlike Joule's heat, which is proportional to the square of current, in this pump, the heat is proportional to the current.

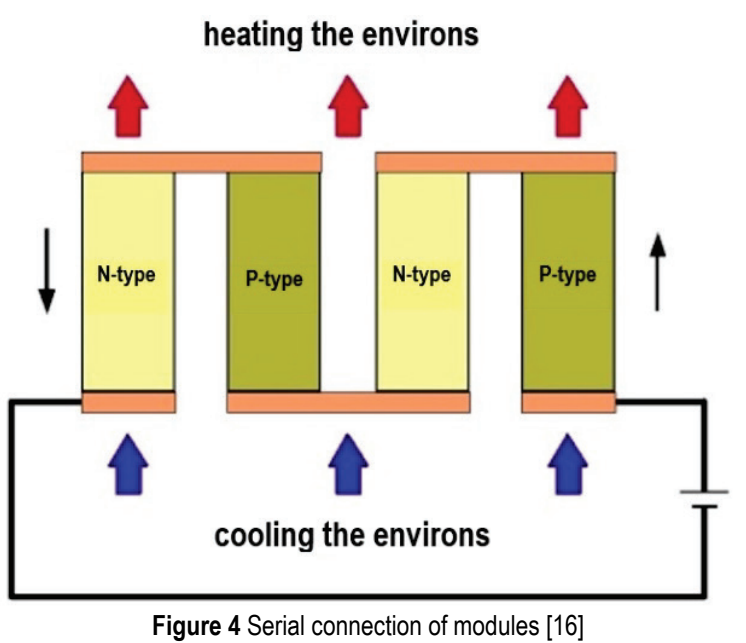

The basic module for the thermoelectric generator is shown in Fig. 5.

If the hotter ends of the p-type and n-type semiconductors are joined together by metal, and electrical resistance is connected between the colder ends, the voltage caused by the Seebeck effect will send the electric current through resistance. Such a joint generates power on the 
electric consumer. The serial connection of more modules can create higher voltages.

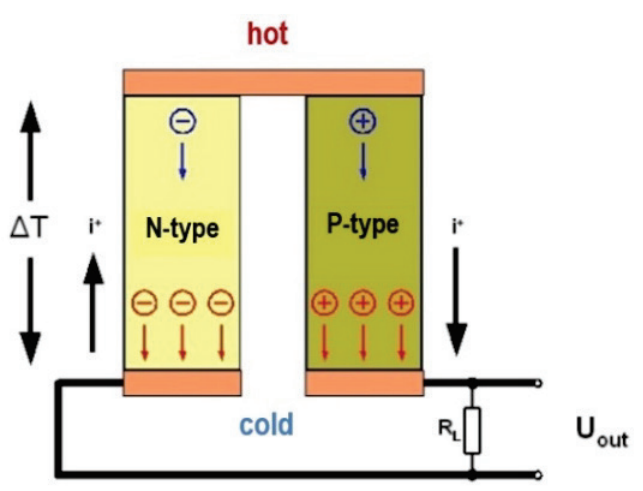

Figure 5 Thermoelectric generator [16]

\section{MATERIALS FOR THERMOELECTRICAL MODULES}

Until the end of the last century, an interest in thermoelectric modules was not large because of low efficiency in most applications. Theoretical analyses suggesting that certain material structures could improve the efficiency of thermoelectric modules stimulated the experimental research of different material structures with the aim of realizing modules that would have acceptable efficacy in certain applications [15-17].

In the realization of thermoelectric modules, high efficiency is important at the transformation of electricity in the heat or vice versa. The most important requirements for module materials are electrical and thermal conductivity. Electrical conductivity should be as large as possible. Considering this, they must be cold at one end of the module and warm at the other, and the heat conductivity should be as low as possible in order to maintain the temperature difference, i.e. the high temperature gradient.

The problem is that materials that have high electrical conductivity also have high thermal conductivity. Therefore, as thermoelectric materials are those semiconductor structures investigated, that show the high conductivity of free electrons and at the same time aggravating the movement of a quantum of heat energy (phonon). Until today, the compounds of the tellurite family have been shown as one of the best.

The materials from which thermoelectric modules were made are called thermoelectrics. Their quality is evaluated by a parameter called the thermoelectric figure of merit $(Z T)$, which is defined by the Eq. (1):

$$
Z T=\frac{\alpha^{2} \sigma}{\kappa} T
$$

Where $\alpha$-Seebeck's coefficient, $\sigma$ - specific conductivity of the material, $\kappa$ - coefficient of thermal conductivity, and $T-$ temperature. The dependency of $Z T$ factors on today's temperature change most frequently used p-types and n-types of thermoelectrics, as it is shown in Figs. 6 and 7, respectively.
For $\mathrm{PbTe}$, the values of the $Z T$ factor in 1960 and today are shown. Significant improvements can be noted, due to technological progress. It is also visible that different materials have maximal $Z T$ at different temperature ranges: lead-telluride ( $\mathrm{PbTe}$ ), silicon-germanium ( $\mathrm{SiGe}$ ) and bismuth antimony alloy (Bi-Sb).

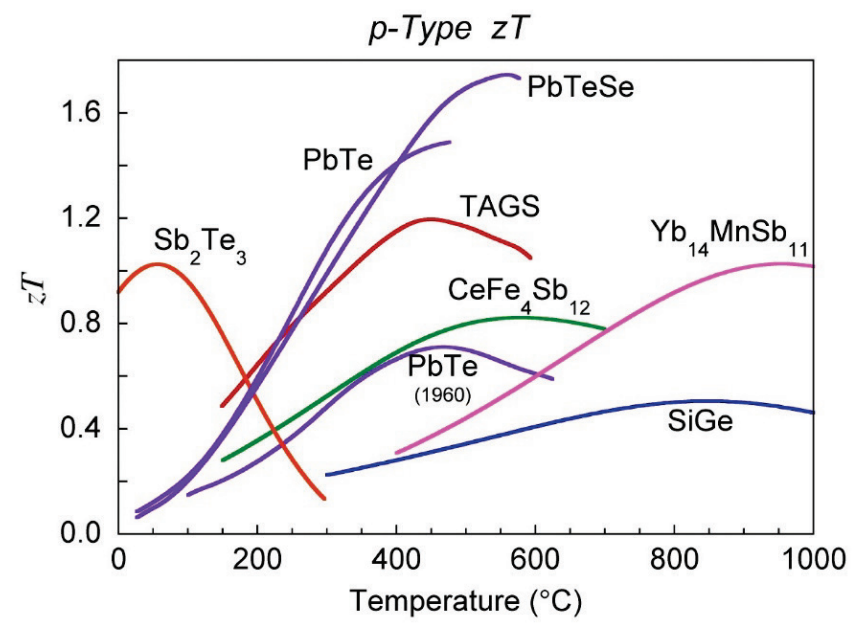

Figure 6 Thermoelectric figure of merit for the $p$-type of thermoelectrics [17]

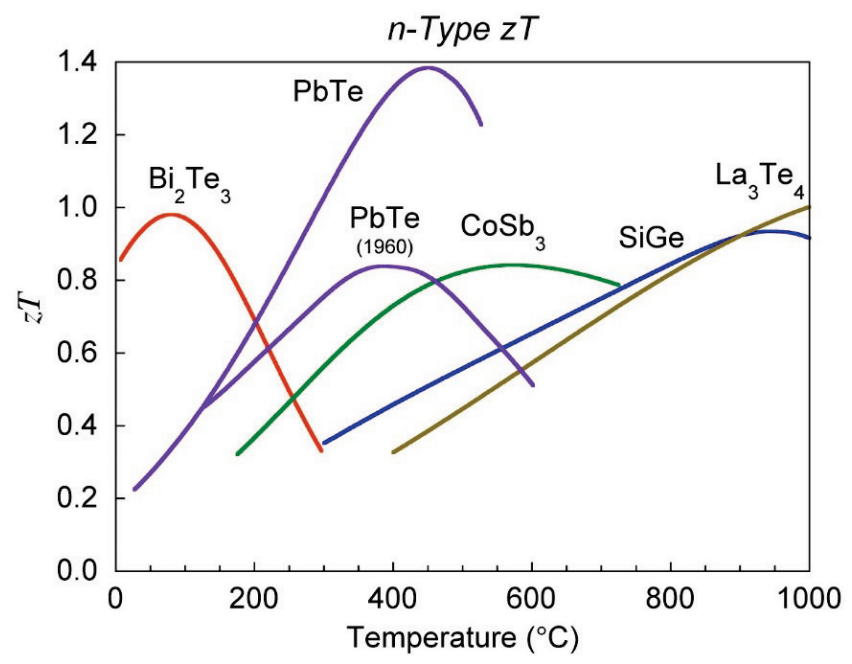

Figure 7 Thermoelectric figure of merit for the n-type of thermoelectrics [17]

The higher the value of the thermoelectric figure of merit, the better the thermoelectric material. Today, most materials have the value of $Z T$ less than 1 . The $Z T$ value of the specific material will be higher if its specific electrical conductivity is higher and if the coefficient of thermal conductivity is lower. For a particular dielectric, the area where $Z T$ is the highest is obtained with well-doped p-type and n-type semiconductors (free concentration of electrons or holes between $10^{19}$ and $10^{20}$ per cubic centimeter).

Today, the intensive search for materials that will have a $Z T$ greater than 1 is conducted by using the latest developments in the field of nanotechnology [16]. Thereby, a particular interest is placed on a complex crystal structure where the aim is to disperse the phonons within the unit cell of the structure, thus reducing thermal conductivity, but without disturbing the flow of electrons, i.e. without reducing 
electrical conductivity. According to the available information, the value of $Z T=2.4$ is reached, and in the near future $Z T=3$ can be expected [13].

Although the effectiveness of new materials has to yet be tested, the promising $Z T$ intensifies the research and experimentation with new thermoelectrics. For a large number of applications, materials with a thermoelectric figure of merit between 2 and 3 will be competitive to other renewable energy sources.

\section{COOLING}

Thermoelectric modules are used for heating or cooling when the electrical current is sent through them, or for electricity generation when one side is heated and the other one is cooled. In both applications, the most important part is the good thermal bondage of the hot and cold side of the module and environment. On the other hand, thermal conductivity within the module itself should be as low as possible in order to maintain a temperature difference of the hot and cold sides.

If the module is used for cooling, the other side of the module will dissipate the heat. Therefore, it is necessary to mount a cooler on the module to dissipate the generated Joule's heat because of the electrical resistance of the module and the heat from the heat pump. The surface of the module that for the beholder appears to be smooth and flat, under the magnifying glass often looks as seen in Figure 8 . When the heat cooler is placed on such a surface, the heat passes only where the surfaces are touching, which is only about $5 \%$ of the surface. The larger parts of the surfaces $(95 \%)$ actually do not touch each other. In order to increase contact surface, a thermal paste must be used to fill the cavities.

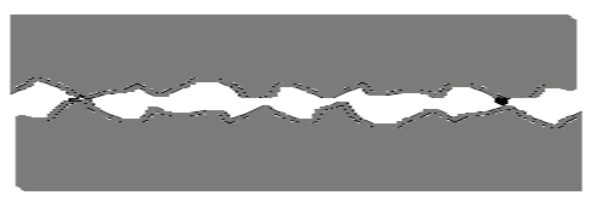

Figure 8 The contact of two apparently smooth surfaces

The cooler should have the temperature increase as low as possible during the operation of the module. The acceptable increase of temperature of the passive cooler is $5-10{ }^{\circ} \mathrm{C}$ above the ambient temperature. For low-power heat pumps, the passive coolers with natural convection of heat resistance of $0.5^{\circ} \mathrm{C} / \mathrm{W}$, but often also with over $10^{\circ} \mathrm{C} / \mathrm{W}$, are used. The coolers combined with a cooling fan (with forced convection) with a heat resistance of $0.02-0.5^{\circ} \mathrm{C} / \mathrm{W}$ are more efficient. The liquid cooled passive coolers have an even higher thermal efficiency per volume unit, of 0.01 to $0.1{ }^{\circ} \mathrm{C} / \mathrm{W}$.

\section{APLICATION OF THERMOELECTRICAL MODULES}

The greatest advantage of thermoelectric modules is that they work without moving parts, which significantly simplifies maintenance. Moreover, their reliable and longlasting operation does not depend on the module position or the environment. They do not create clamor or electric noise during operation, so they can be used together with sensitive electronic sensors. Compared to mechanical systems for the same purpose, thermoelectric modules are much smaller and lighter, having the same power. Additionally, they do not use any chemicals or gases that can be harmful to the environment during the work.

Thermoelectric modules are used in activities with different technical requirements: military, medical, industry, telecommunications, science and consumer technology. The heating or cooling function depends only on the polarity of the connected voltage.

Thermoelectric refrigerators can remove the heat from $\mathrm{mW}$ to several thousands of $\mathrm{W}$. The most used modules are those with the size of $100 \mathrm{~W}$.

Thermoelectric modules are used as coolers for laser diodes, laboratory instruments, electronic casings and components, etc. With a suitable control circuit, the temperature adjustment precision can be even below \pm 0.001 ${ }^{\circ} \mathrm{C}$.

The function of generating the electricity depends on the temperature difference between the hot and cold side of the module.

Today, many companies produce thermoelectric generators that convert waste heat into electricity. They use different heat sources (hot plate heaters, gas cookers, wood stoves, etc.) that can generate power from about $10 \mathrm{~W}$ to the size of several hundreds of W. For cooling, they use ambient temperature, fan or water. Thermoelectric generators are mostly used for lighting, powering up the electronic devices and charging batteries. They are lightweight, small in dimensions, robust and practical to carry.

The most modern thermoelectrics allow the realization of thermoelectric generators that are by volume competitive to batteries and to solar cells and wind power plants by economics.

Because of their robustness, (lifetime of modules is often higher than the lifetime of other equipment), they are interesting for the military industry and space programs (spacecraft, satellites) and for applications in inaccessible locations or in extreme conditions (lighthouses, isolated islands).

At remote, isolated locations, robust thermoelectric generators that use waste heat can be combined with a solar power plant as the reliable power supply system for various consumers (sensors, actuators, wireless communication devices, maintenance and monitoring systems, etc.).

In the area of small dimensions, low power and small temperature differences, thermoelectric generators are particularly interesting in converting the heat of a human body into electrical energy. An example described in [18] is a wristwatch powered by a thermoelectric generator with $22 \mu \mathrm{W}$ at a temperature difference of $1.5^{\circ} \mathrm{C}$.

Thermoelectric generators, due to their robustness and longevity, can provide power supplies for medical devices such as pacemakers. That can extend life expectancy for thirty years, a fivefold increase compared to today's technology. 


\section{TESTINGS}

Testing was conducted in order to establish the possibilities of application of commercially available modules for electricity generation from the waste heat of some machines.

To draw the conclusions on the possibility of using thermoelectric generators, three different modules of M1, M2 and M3 were purchased. The dimensions of the modules are:

- M1: length $40 \mathrm{~mm}$, width $40 \mathrm{~mm}$, height $3.6 \mathrm{~mm}$,

- M2: length $40 \mathrm{~mm}$, width $40 \mathrm{~mm}$, height $3.8 \mathrm{~mm}$,

- $\mathrm{M} 3$ : length $57 \mathrm{~mm}$, width $54.4 \mathrm{~mm}$, height $3.4 \mathrm{~mm}$.

Module prices are also different. Module M1 is the cheapest, M2 is considerably more expensive and M3 is even more expensive. Identical tests were conducted on all three modules, under the same conditions. The aim was not to measure module parameters in laboratory conditions, but to compare the modules' properties by using the available equipment.

The aluminum plates, each $10 \mathrm{~mm}$ thick, were placed on both sides of the thermoelectric module. To reduce the thermal resistance between the aluminum plates and the modules, a thin layer of thermal paste was applied on both sides of the module. The aluminum plate on the warm side was heated with electric heaters and the plate on the cold side was cooled with ice. Holes were made in aluminum plates at the contact place with the module, within which the temperature sensors were installed. During the measurement process, it was noticed that the quality of the join with the ice tray on the cold side of the module had the most influence on the generated voltage.

For each module, the following measurements were done, according to the electrical schemes in Figs. 9-11:

1. Idle voltage at various temperature differences (Fig. 9),

2. Short circuit current at various temperature differences (Fig. 10),

3. Currents and voltages for various loads on the module at various temperature differences (Fig. 11).

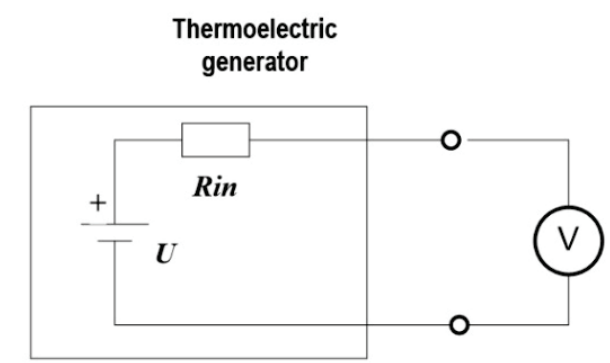

Figure 9 Electrical scheme for the measurement of idle voltage

Idle voltage was measured with a voltmeter connected to the external outputs of thermogenerators. The hot side of the M1 module was heated up to $120^{\circ} \mathrm{C}$, and of the modules M2 and $\mathrm{M} 3$ up to $180^{\circ} \mathrm{C}$, whilst cooling the other side with ice. The temperature of the cold side $\left(T_{c}\right)$ did not exceed $30^{\circ} \mathrm{C}$ at $\mathrm{M} 1,40{ }^{\circ} \mathrm{C}$ at $\mathrm{M} 2$ and $50{ }^{\circ} \mathrm{C}$ at $\mathrm{M} 3$, even at the highest temperatures of the warm side $\left(T_{h}\right)$. At the warm side of the modules, the permissible temperature limits were considered (for M1, $T_{h}$ did not exceed $120{ }^{\circ} \mathrm{C}$, and for M2 and M3 190 $\left.{ }^{\circ} \mathrm{C}\right)$. It should be noted that the same temperature difference $\left(\Delta T=T_{h}-T_{c}\right)$ can be achieved with e.g. $T_{c}=20^{\circ} \mathrm{C}$ or $T_{c}=80$

${ }^{\circ} \mathrm{C}$. Higher $T_{c}$ results in reduced power for the same load.

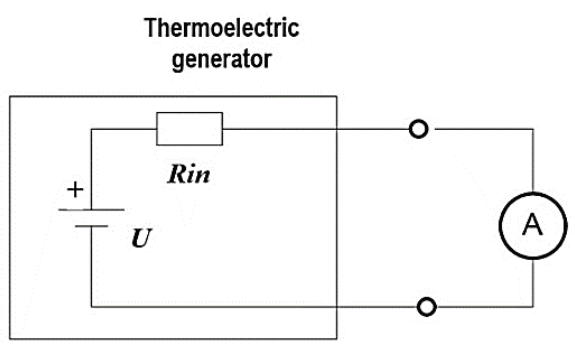

Figure 10 Electrical scheme for the measurement of short circuit current

\section{Thermoelectric generator}

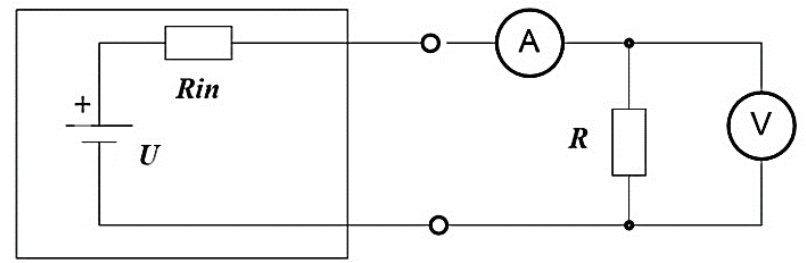

Figure 11 Electrical scheme for the measurement of currents and voltages for various loads

In Fig. 12, it can be seen that the module M1 reached the maximum idle voltage of around $4 \mathrm{~V}, \mathrm{M} 2$ around $10 \mathrm{~V}$ and $\mathrm{M} 3$ around $18 \mathrm{~V}$.

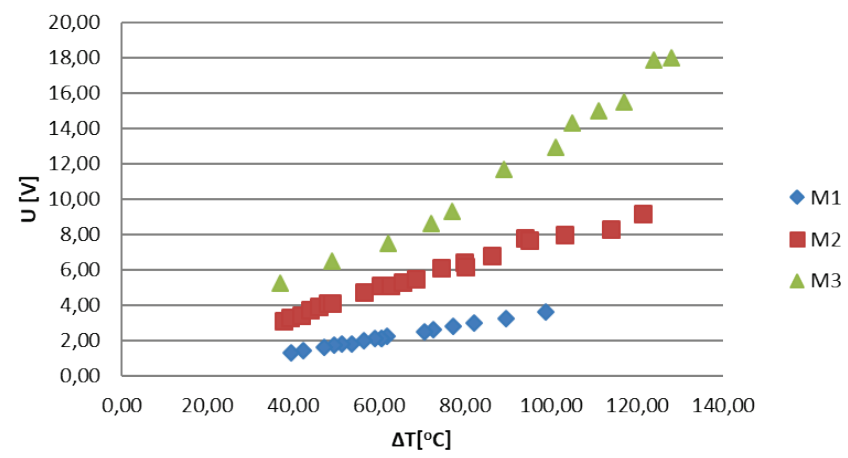

Figure 12 Dependency of the idle voltage and the temperature difference

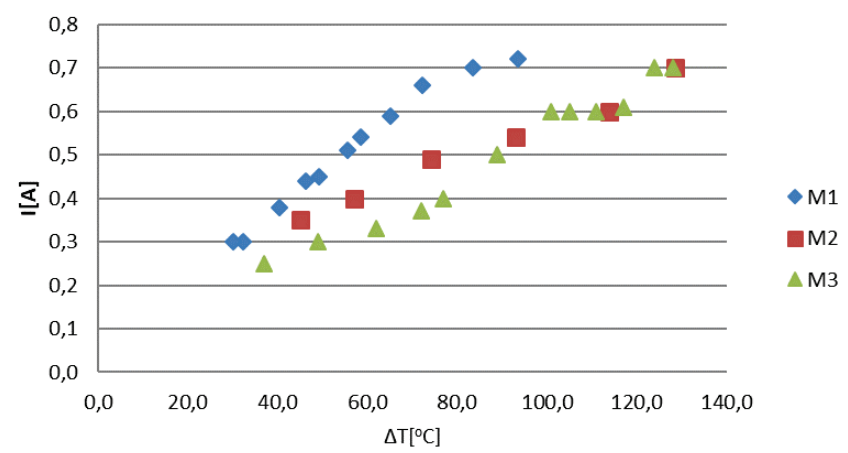

Figure 13 Dependency of the short circuit current and the temperature difference 
The short-circuit current was measured with an ammeter connected to the external outputs of thermogenerators. The measurement procedure was carried out for different temperatures under the same conditions as previously explained. Fig. 13 shows the obtained results. It can be noticed that the maximum short circuit current is approximately the same for all modules despite the different idling voltages. The reason for that is the greater internal resistance of the M2 and M3 modules.

Based on the short circuit current measured according to Fig. 10 and idle voltage measured according to Fig. 9, the internal resistance of the thermogenerator as a source can be obtained $\left(R_{i n}=U / I\right)$. The changes of $R_{i n}$ with the change of temperature difference is shown in Fig. 14.

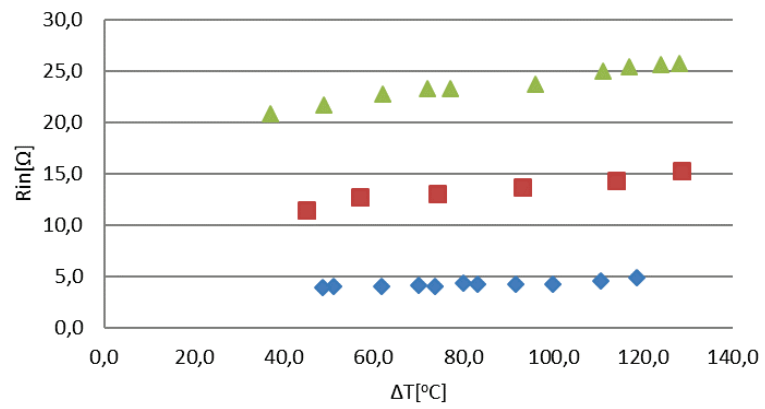

M1

$\square \mathrm{M} 2$

$\triangle \mathrm{M} 3$

Figure 14 Dependency of the internal resistance and the temperature difference

The thermogenerator M1 has an internal resistance of around $5 \Omega$, M2 of around $15 \Omega$ and M3 of $20-30 \Omega$. It is visible that the internal resistance of the thermo-generator slightly increases with the increase of $\Delta T$.

Power measurement as the product of the output current and voltage $(P=U \cdot I)$ was performed on resistors of $4 \Omega, 10$ $\Omega, 20 \Omega$ and $40 \Omega$, according to the electrical scheme in Fig. 11. Figs. 15-18 show the achieved power for different resistors for all three modules.

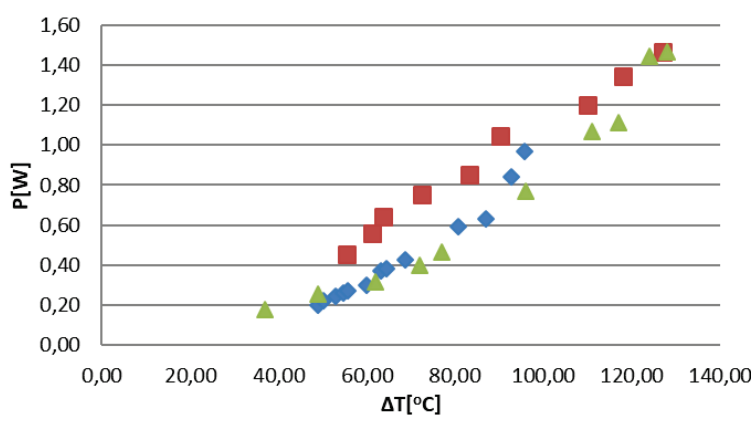

Figure 15 Achieved power for all three modules at $R=4 \Omega$

Based on the diagrams in Figs. 15-18, it can be concluded which module will give the maximum power for a given temperature difference, with which resistance, to a certain consumer. Thus, the module M1 gives the highest power rating to the resistor of $4 \Omega$, the module M2 to the resistor of $10-20 \Omega$ and the module M3 to the resistor around $20 \Omega$. Figs. 19-21 show the power that each module gives for different loads. From these diagrams, the expected useful power on the resistors can be determined for a given temperature difference.

The efficiency of the tested modules of M1, M2 and M3 is below $10 \%$ and it depends on the connected load. For the tested loads, it was mostly higher on M3 and M2, while slightly smaller on M1. Modules that are more expensive are more efficient, because they can work at higher temperature differences.

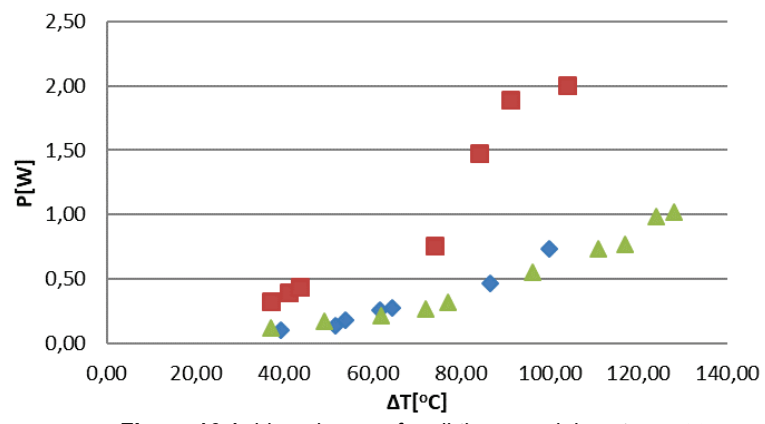

$\bullet \mathrm{M} 1$

$\mathrm{M} 2$

$\triangle \mathrm{M} 3$

Figure 16 Achieved power for all three modules at $R=10 \Omega$

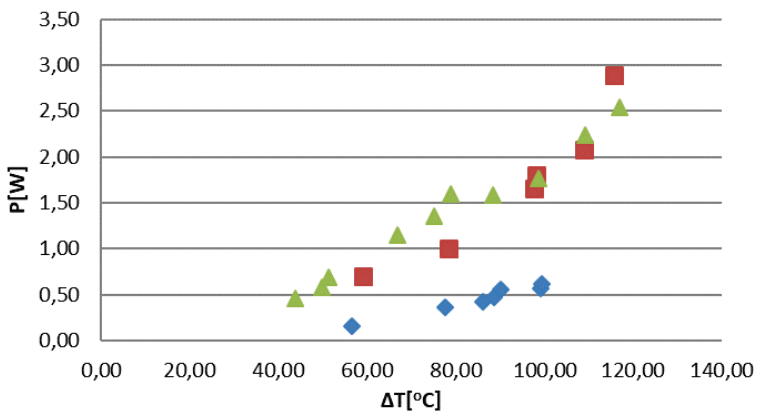

Figure 17 Achieved power for all three modules at $R=20 \Omega$

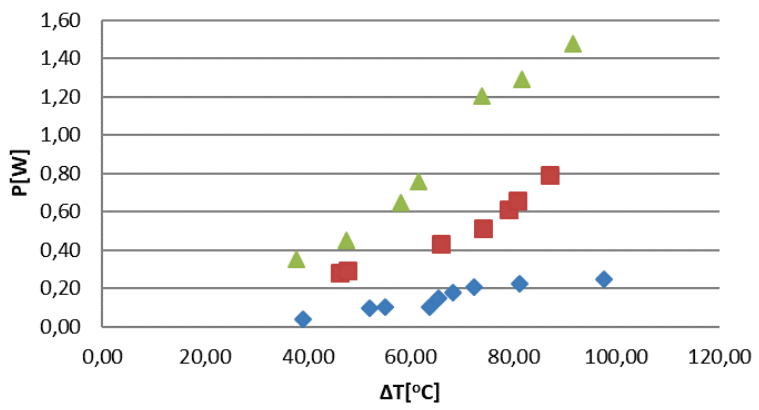

Figure 18 Achieved power for all three modules at $R=40 \Omega$

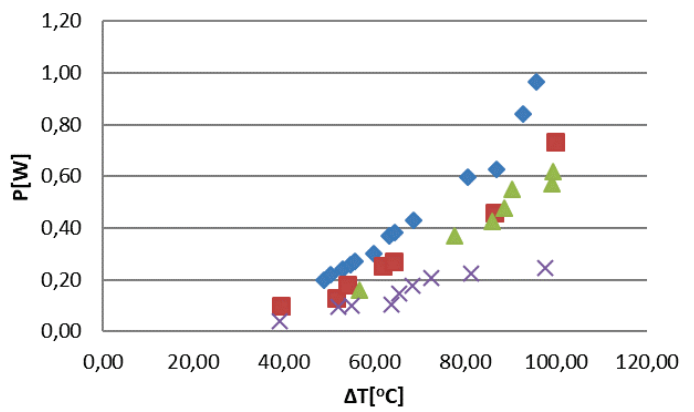

M1-40hm M1-10ohm $\triangle$ M1-20ohm $\times$ M1-40ohm

Figure 19 Achieved power for the module M1 for each resistor 

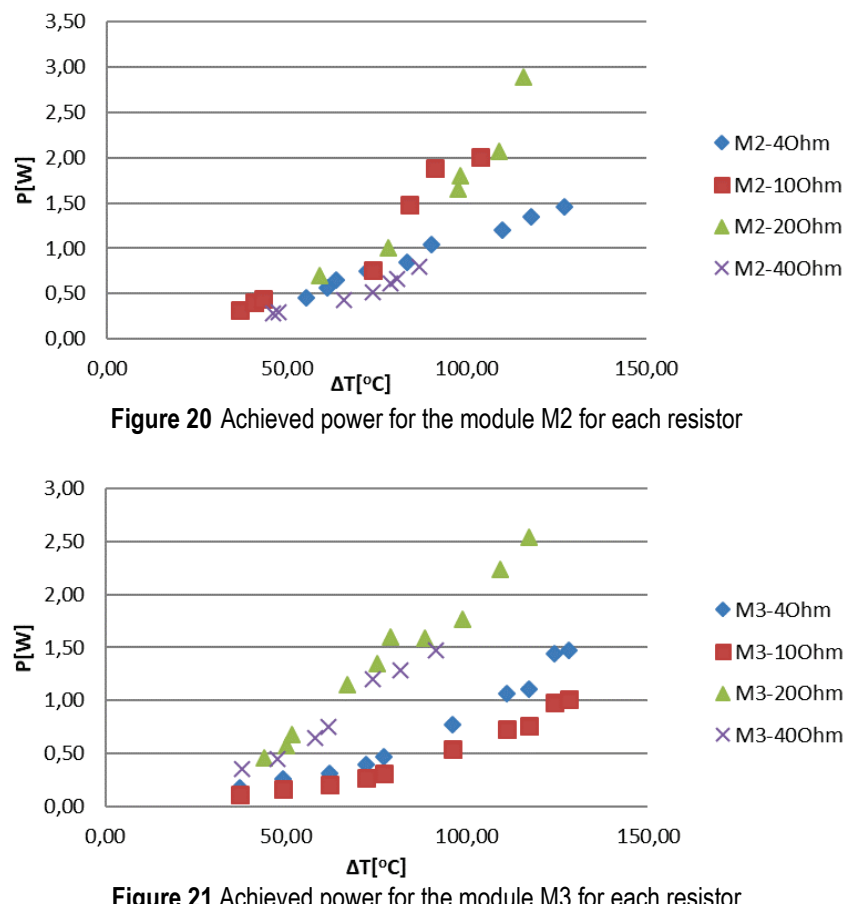

\section{CONCLUSION}

Although the efficiency of the thermoelectric modules is still quite low, the latest research has resulted in materials needed for the building of increasingly more efficient modules. Those modules can be competitive to the renewable sources of electricity such as solar power plants and wind power plants. The greatest advantages of thermoelectric generators are small dimensions, compactness, long lasting, quiet and reliable operation because they do not have moving parts, they have low maintenance requirements and a wide range of heat sources.

Despite the low efficiency of the conversion of thermal energy to electricity, the interest in thermoelectric modules is constantly increasing due to the fact that waste heat is used and the thermoelectric figure of merit of the thermoelectrics increases. Therefore, the presence of thermoelectric modules and thermoelectric generators in the market is on the rise. Moreover, the generators used today have the power range from $\mu \mathrm{W}$ to $\mathrm{kW}$.

This paper presents the experimental results of measurements on three different modules. The maximum voltage of $3.6 \mathrm{~V}$ at $\Delta T=100^{\circ} \mathrm{C}$ was achieved by measuring on module $\mathrm{M} 1$. At the same value of $\Delta T, \mathrm{M} 2$ gives $8 \mathrm{~V}$ and M3 gives $13 \mathrm{~V}$. Since the modules of M2 and M3 can work at higher temperatures, the measured voltage on M2 is $9.2 \mathrm{~V}$ and on $\mathrm{M} 3$ it is $18 \mathrm{~V}$ at a temperature difference of $\Delta T=120$ ${ }^{\circ} \mathrm{C}$. All modules show linear dependence of the generated voltage on the rise in the temperature difference $\Delta T$ (Fig. 9). The maximum short circuit current is approximately the same on all modules, considering the fact that the modules that can generate higher voltages have higher internal resistance (Fig. 11). The maximum power measured on the module $M 1$ is about $1 \mathrm{~W}$ at $\Delta T=100^{\circ} \mathrm{C}$, and for M2 and M3 slightly above $2.5 \mathrm{~W}$ at $\Delta T=120^{\circ} \mathrm{C}$.
The tested modules point to the conclusion that the modules that are more expensive give higher energy density per unit of volume at higher temperatures. On the other side, cheaper modules are more cost-effective due to a large number of inexpensive modules in order to compensate for the lack of power on a single unit. Considering the small dimensions of the modules, the realization of a thermal power generator with lower $\mathrm{kW}$ power can be competitive with wind power plants and solar power plants.

Note: This research was partially presented as a poster at the International Conference MATRIB 2017 (29 June - 2 July 2017, Vela Luka, Croatia).

\section{REFERENCES}

[1] Nolas, G. S., Sharp, J., \& Goldsmid, H. J. (2001). Thermoelectrics: basic principles and new materials developments, Springer. https://doi.org/10.1007/978-3-662-04569-5

[2] Zheng, X. F., Liu, C. X., Yan, Y. Y., \& Wang, Q. (2014). A review of thermoelectrics research - Recent developments and potentials for sustainable and renewable energy applications. Renewable and Sustainable Energy Reviews, 32, 486-503. https://doi.org/10.1016/j.rser.2013.12.053

[3] Chen, J., Li, K., Liu, C., Li, M., Lv, Y., Jia, L., \& Jiang, S. (2017). Enhanced Efficiency of Thermoelectric Generator by Optimizing Mechanical and Electrical Structures. Energies, 10, 1329. https://doi.org/10.3390/en10091329

[4] Liu, C., Chen, P., \& Li, K. (2014). A $1 \mathrm{~kW}$ Thermoelectric Generator for Low-temperature Geothermal Resources. Proceedings, Thirty-Ninth Workshop on Geothermal Reservoir Engineering, Stanford University, Stanford, California, February 24-26,

[5] LeBlanc, S. (2014). Thermoelectric generators: Linking material properties and systems engineering for waste heat recovery applications. Sustainable Materials and Technologies, 1-2, 26-35. https://doi.org/10.1016/j.susmat.2014.11.002

[6] Fairbanks, J. W. (2006). Thermoelectric developments for vehicular applications. Diesel Engine-Efficiency and Emissions Research (DEER) Conference, Detroit, MI.

[7] Lui, X., Deng, Y. D., Chen, S., Wang, W. S., Xu, Y. \& Su, C. Q. (2014). A case study on compatibility of automotive exhaust thermoelectric generation system, catalytic converter and muffler. Case Studies in Thermal Engineering, 2, 62-66. https://doi.org/10.1016/j.csite.2014.01.002

[8] Su, C. Q., Wang, W. S., Liu, X., \& Deng, Y. D. (2014). Simulation and experimental study on thermal optimization of the heat exchanger for automotive exhaust-based thermoelectrical generators. Case Studies in Thermal Engineering, 4, 85-91. https://doi.org/10.1016/j.csite.2014.06.002

[9] Bai, S., Lu, H., Wu, T., Yin, X., Shi, X., \& Chen, L. (2014). Numerical and experimental analysis for exhaust heat exchanger for automobile thermoelectric generators. Case Studies in Thermal Engineering, 4, 99-112. https://doi.org/10.1016/j.csite.2014.07.003

[10] Orr, B., Akbarzadeh, A., Mochizuki, M., \& Singh, R. (2016). A review of car waste heat recovery systems utilising thermoelectric generators and heat pipes. Applied Thermal Engineering, 101, 490-495.

https://doi.org/10.1016/j.applthermaleng.2015.10.081 
[11] Jaworski, M., Bednarczyk, M., \& Czachor, M. (2016). Experimental investigation of thermoelectric generator (TEG) with PCM module. Applied Thermal Engineering, 96, 527-533. https://doi.org/10.1016/j.applthermaleng.2015.12.005

[12] Brown, S. R., Kauzlarich, S. M., Gascoin, F., \& Snyder, G. J. (2006). Yb $b_{14} \mathrm{MnSb}_{11}$ : New High Efficiency Thermoelectric Material for Power Generation. Chemistry of Materials, 18(7), 1873-1877. https://doi.org/10.1021/cm060261t

[13] Yan, X., Poudel, B., Ma, Y., Liu, W. S., Joshi, G., Wang, H., Lan, Y., Wang, D., Chen, G., \& Ren, Z. F. (2010). Experimental Studies on Anisotropic Thermoelectric Properties and Structures of n-Type $\mathrm{Bi}_{2} \mathrm{Te}_{2.7} \mathrm{Se}_{0.3}$. Nano Letters, 10(9), 3373-3378. https://doi.org/10.1021/nl101156v

[14] Cooley, J. A., Promkhan, P., Gangopadhyay, S., Donadio, D., Pickett, W. E., Ortiz, B. R., Toberer, E. S., \& Kauzlarich, S. M. (2018). High Seebeck Coefficient and Unusually Low Thermal Conductivity near Ambient Temperatures in Layered Compound $\mathrm{Yb}_{2-\mathrm{x}} \mathrm{Eu}_{\mathrm{x}} \mathrm{CdSb}_{2}$. Chemistry of Materials, 30(2), 484-493. https://doi.org/10.1021/acs.chemmater.7b04517

[15] Qiu, B., Bao, H., Zhang, G., Wu, Y., \& Ruan, X. (2012). Molecular dynamics simulations of lattice thermal conductivity and spectral phonon mean free path of PbTe: Bulk and nanostructures. Computational Materials Science, 53, 278285. https://doi.org/10.1016/j.commatsci.2011.08.016

[16] Šumiga, I., Grđan, M., \& Huđek J. (2007). Termoelektrični moduli - fizikalne osnove i smjernice za uporabu. Technical journal, 1(1-2), 5-10.

[17] http://thermoelectrics.matsci.northwestern.edu/thermoelectrics Available May 2017.

[18] Snyder, G. J. (2008). Small thermoelectric generators. Electrochemical Society Interface, 17, 54-56.

\author{
Authors' contacts: \\ Ivan ŠUMIGA, MSc, Senior Lecturer \\ University North, University Centre of Varaždin \\ 104. brigade 3, 42000 Varaždin, Croatia \\ e-mail: ivan.sumiga@unin.hr \\ Dunja SRPAK, PhD, Lecturer \\ (Corresponding author) \\ University North, University Centre of Varaždin \\ 104. brigade 3, 42000 Varaždin, Croatia \\ e-mail: dunja.srpak@unin.hr
}

Živko KONDIĆ, PhD, Professor

University North, University Centre of Varaždin

104. brigade 3, 42000 Varaždin, Croatia

e-mail: zivko.kondic@unin.hr 